\section{Sporadic Creutzfeldt-Jakob disease with focal findings: caveats to current diagnostic criteria}

\author{
Edward C. Mader Jr., Rima El-Abassi, \\ Nicole R. Villemarette-Pittman, \\ Lenay Santana-Gould, \\ Piotr W. Olejniczak, John D. England \\ Department of Neurology, Louisiana \\ State University Health Sciences Center, \\ New Orleans, LA, USA
}

\section{Abstract}

The clinical diagnosis of Creutzfeldt-Jakob disease (CJD) is largely based on the 1998 World Health Organization diagnostic criteria. Unfortunately, rigid compliance with these criteria may result in failure to recognize sporadic CJD (sCJD), especially early in its course when focal findings predominate and traditional red flags are not yet present. A 61-yearold man presented with a 3-week history of epilepsia partialis continua (jerking of the left upper extremity) and a 2-week history of forgetfulness and left hemiparesis; left hemisensory neglect was also detected on admission. Repeated brain magnetic resonance imaging (MRI) showed areas of restricted diffusion in the cerebral cortex, initially on the right but later spreading to the left. Electroencephalography (EEG) on hospital days 7, 10, and 14 showed right-sided periodic lateralized epileptiform discharges. On day 20, the EEG showed periodic sharp wave complexes leading to a diagnosis of probable sCJD and subsequently to definite sCJD with brain biopsy. Neurological decline was relatively fast with generalized myoclonus and akinetic mutism developing within 7 weeks from the onset of illness. CJD was not immediately recognized because of the patient's focal/lateralized manifestations. Focal/lateralized clinical, EEG, and MRI findings are not uncommon in SCJD and EEG/MRI results may not be diagnostic in the early stages of sCJD. Familiarity with these caveats and with the most current criteria for diagnosing probable sCJD (University of California San Francisco 2007, MRI-CJD Consortium 2009) will enhance the ability to recognize sCJD and implement early safety measures.

\section{Introduction}

Creutzfeldt-Jakob disease (CJD) belongs to a family of rare transmissible brain disorders (prion diseases) in which the causative agent (prion) induces abnormal folding of normal cellular proteins (prion proteins) leading to progressive spongiform degeneration and loss of brain neurons (spongiform encephalopathy). Sporadic CJD (sCJD) accounts for $85 \%$ of CJD cases; the remaining $15 \%$ are familial or iatrogenic. Clinically, SCJD is diagnosed based on criteria published by the World Health Organization (WHO) consultation group in 1998.1 These criteria were updated in 2007 by the University of California San Francisco (UCSF) group and in 2009 by the MRI-CJD Consortium (MCC).2,3 The definitive diagnosis of $\mathrm{SCJD}$ requires pathological studies of the brain. 4

Table 1 outlines the WHO, UCSF, and MCC diagnostic criteria for probable/possible sCJD.1-3 Diagnosing probable sCJD requires the presence of clinical manifestations and at least one positive laboratory finding. Both WHO and UCSF criteria require the presence of unexplained cognitive decline, at least two other neurological disturbances, and at least one positive laboratory test finding. The MCC criteria require at least two neurological disturbances (cognitive decline may or may not be one of them) and at least one positive laboratory test finding. Based on WHO or MCC criteria, a diagnosis of possible sCJD is made if the patient has the clinical signs required to diagnose probable sCJD, but none of the laboratory signs are present. Possible SCJD is not a diagnostic entity in the UCSF criteria set.

In theory, replacing the WHO criteria with the UCSF/MCC criteria will improve diagnostic yield. Nevertheless, sCJD with prominent focal manifestations and/or non-diagnostic electroencephalography/magnetic resonance imaging (EEG/MRI) findings can still escape diagnosis no matter which criteria set is used. We present a case of sCJD that was not immediately suspected because of the focal/lateralized clinical, EEG, and MRI manifestations.

\section{Case Report}

A 61-year-old man presented with a 3-week history of left upper extremity jerking and a 2week history of left hemiparesis, forgetfulness, and inattention. On admission, the patient was alert and oriented to self and place, but not to time; his vital signs were normal. He manifested left arm clonic movements consistent with epilepsia partialis continua (EPC); this was easily suppressed with lorazepam. The neurological exam revealed $3 / 5$ strength in the distal left upper extremity muscles, $4 / 5$ strength in the left lower extremity muscles, +3 reflexes on the left and +2 on the right, left Babinski sign, and left hemisensory neglect. Primary
Correspondence: Edward C. Mader, Department of Neurology, Louisiana State University Health Sciences Center, 1542 Tulane Avenue, Rm 763, New Orleans, LA 70112, USA.

E-mail: emader@lsuhsc.edu

Key words: Creutzfeldt-Jakob disease, sporadic CJD, EEG, periodic sharp wave complexes, epilepsia partialis continua, periodic lateralized epileptiform discharges, MRI.

Conflict of interests: the authors declare no potential conflict of interests.

Contributions: ECM, REA, LSG, conceived the idea and prepared the manuscript. REA was the consulting neurology resident for this case; NVP, PWO, JDE, provided a detailed review of the content and structure of the manuscript resulting in significant changes to the original document.

Acknowledgments: we are grateful to all individuals and parties of the Ochsner Medical Center, especially to Dr. Eugene Ramsay and Dr. Frank Oser, III, whose combined effort and expertise resulted in a definitive diagnosis of CreutzfeldtJakob disease.

Received for publication: 7 August 2012.

Revision received: 18 September 2012.

Accepted for publication: 17 November 2012.

This work is licensed under a Creative Commons Attribution NonCommercial 3.0 License (CC BYNC 3.0).

(C) Copyright E.C. Mader Jr et al., 2012

Licensee PAGEPress, Italy

Neurology International 2013; 5:e1

doi:10.4081/ni.2013.el

sensation, cranial nerves, and cerebellar function were intact. Because of the foca/lateralized findings and the patient's history of hypertension, hyperlipidemia, myocardial infarction, and coronary artery bypass graft surgery, stroke was diagnosed on admission.

Brain MRI on hospital days 1, 7, and 20 showed restricted diffusion in the cerebral cortex; the cortical areas affected appeared hyperintense on diffusion-weighted imaging (DWI) and hypointense on apparent diffusion coefficient (ADC) (Figure 1). Some cortical lesions were also visible on fluid attenuation inversion recovery (FLAIR) sequences. At first limited to the right occipitoparietal, right posterior frontal, and bilateral mesial frontal cortices, the areas of restricted diffusion expanded and spread to the adjacent cortices in the right, and eventually, in the left hemisphere. Restricted diffusion was not present in the basal ganglia and thalamus.

Scalp EEG on hospital days 7, 10, and 14 showed right hemispheric periodic lateralized epileptiform discharges (PLEDs) in the form of sharp and slow waves with a right centropari- 
Table 1. Clinical diagnostic criteria for sporadic Creutzfeldt-Jakob disease (sCJD). This table outlines the World Health Organization (1998), University of California San Francisco (2007), and MRI-CJD Consortium (2008) criteria for diagnosing probable sCJD and possible sCJD. Brain biopsy is required for premortem diagnosis of definite $\mathrm{sCJD}$.

\section{World Health Organization diagnostic criteria (1998) \\ Probable $\mathrm{sCJD}=\mathrm{A}+$ at least 2 of $\mathrm{B}+$ at least 1 of $\mathrm{C}$ \\ Possible $\mathrm{sCJD}=\mathrm{A}+$ at least 2 of $\mathrm{B}+$ duration $<2$ years}

A. Progressive dementia

B. Specific neurological manifestations

1. Myoclonus

2. Visual or cerebellar disturbance

3. Pyramidal or extrapyramidal dysfunction

4. Akinetic mutism

C. Laboratory tests

1. Positive EEG: periodic sharp wave complexes

2. Positive CSF: 14-3-3 protein

D. Routine investigations should not suggest an alternative diagnosis

\section{University of California San Francisco diagnostic criteria (2007) \\ Probable $\mathrm{s} C \mathrm{JD}=\mathrm{A}+$ at least 2 of $\mathrm{B}+$ at least 1 of $\mathrm{C}+\mathrm{D}$ \\ Possible sCJD is not defined in this criteria set}

A. Rapid cognitive decline

B. Specific neurological manifestations

1. Myoclonus

2. Pyramidal or extrapyramidal

3. Visual

4. Cerebellar

5. Akinetic mutism

6. Other focal higher cortical sign (e.g., neglect, aphasia, apraxia, acalculia)

C. Laboratory tests

1. Positive EEG: periodic epileptiform discharges

2. Positive MRI: subcortical or cortical gyral hyperintensity (cortical ribboning) on DWI and preferably restricted diffusion on $\mathrm{ADC}$ map

D. Routine investigations do not suggest an alternative diagnosis

MRI-CJD Consortium diagnostic criteria (2009)
Probable sCJD = at least 2 of $\mathrm{A}+$ at least 1 of $\mathrm{B}$
Possible sCJD = at least 2 of $\mathrm{A}+$ duration $<2$ years

A. Clinical signs

1. Dementia

2. Cerebellar or visual

3. Pyramidal or extrapyramidal

4. Akinetic mutism

B. Laboratory tests

1. Positive EEG: periodic sharp wave complexes

2. Positive CSF: 14-3-3 protein in patients with a disease duration of less than 2 years

3. Positive MRI: high signal abnormalities in caudate nucleus and putamen or at least two cortical regions (temporal-parietal-occipital) either on DWI or FLAIR

sCJD, sporadic Creutzfeldt-Jakob disease; EEG, electroencephalography; CSF, Cerebrospinal fluid; MRI, magnetic resonance imaging; DWI, diffusion-weighted imaging; $\mathrm{ADC}$, apparent diffusion coefficient. etal maximum and a discharge rate of about 1/second (Figure 2). Some sharp waves were time-locked to the patient's myoclonic jerks. The background EEG was slow and attenuated on both sides. The PLEDs increased in amplitude and became broader in distribution. On day 20, the EEG showed bihemispheric periodic sharp wave complexes (PSWCs) alerting the physicians to the possibility of sCJD.

Cerebrospinal fluid (CSF) analysis showed elevated neuron-specific enolase $(79 \mathrm{ng} / \mathrm{mL})$ but normal glucose, protein, and cell counts. Common and rare causes of infectious and autoimmune encephalitis were excluded including arboviruses, herpes simplex virus, human immunodeficiency virus, paraneoplastic disorders, and Hashimoto's disease. The diagnosis remained elusive until PSWCs appeared suggesting sCJD. CSF was sent out for protein 14-3-3 determination but the specimen was lost in transit. Brain biopsy was performed and pathological studies at Mayo Clinic confirmed sCJD.

Myoclonus was suppressed with valproic acid, levetiracetam, and repeated administration of lorazepam. The patient was also treated empirically with antibiotics, acyclovir, and methylprednisolone. Neurological decline was relatively fast with the patient developing ataxia, nystagmus, generalized myoclonus, and akinetic mutism within 7 weeks after the onset of symptoms. He was discharged to hospice where he could be with family and friends.

\section{Discussion and Conclusions}

In the SCJD case presented, mild cognitive dysfunction was overshadowed by focal/ateralized findings, including EPC, left hemiparesis, left hemisensory neglect, and PLEDs. Such foca/lateralized findings steered the diagnosis

\section{Table 2. Recognizing sporadic Creutzfeldt-Jakob disease in its early stages: important caveats to current criteria.}

\section{Criteria}

1 Rapid cognitive decline and myoclonus occur in a variety of brain disorders and are not specific for sporadic sCJD. In the early stages of sCJD, myoclonus may be absent and cognitive decline may be subtle. Cognitive decline may also be present without myoclonus and myoclonus may be present without evidence of cognitive impairment.

2 Focal/lateralized neurological manifestations are not uncommon in SCJD. In the early stages of sCJD, focal signs/symptoms may confuse the diagnosis, especially if cognitive impairment and myoclonus are not yet evident. The focal signs/symptoms may also be abrupt in onset and mimic a stroke.

3 Epilepsia partialis continua (EPC), which is synonymous with focal myoclonic status epilepticus, should be counted as myoclonus. Failing to will delay the diagnosis of SCJD. The evolution of EPC to bilateral or multifocal myoclonus can increase one's confidence in diagnosing sCJD

4 Scalp electroencephalography (EEG) may show periodic lateralized epileptiform discharges (PLEDs) days/weeks before the appearance of characteristic periodic sharp wave complexes (PSWCs). The PLEDs may or may not be associated with myoclonus/EPC. Serial EEG (e.g. every 5-7 days) may demonstrate the evolution of PLEDs towards PSWCs and increase one's confidence in diagnosing SCJD.

5 Cerebrospinal fluid (CSF) 14-3-3 protein assay has a relatively low specificity and a modest sensitivity for diagnosing sCJD. However, it can still be useful if a patient is suspected of having SCJD whilst the diagnosis of SCJD remains uncertain.

6 Magnetic resonance imaging (MRI), particularly diffusion-weighted imaging (DWI) with apparent diffusion coefficient (ADC) mapping, is proving to be very useful in the early diagnosis of sCJD. The sCJD lesions appear as areas of restricted diffusion (DWI hyperintensities/ADC hypointensities) in the cerebral cortex, basal ganglia, and/or thalamus. Serial MRI (e.g. after 7-14 days) may show the spread of the gray matter lesions and increase one's confidence in diagnosing SCJD.

sCJD, sporadic Creutzfeldt-Jakob disease. 


\section{DWI}

ADC

FLAIR

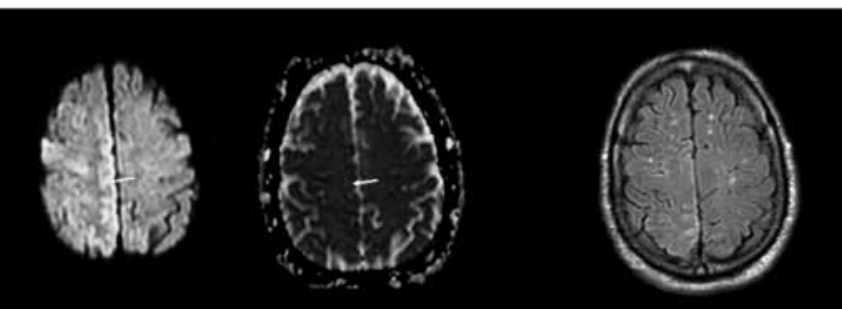

DAY 1
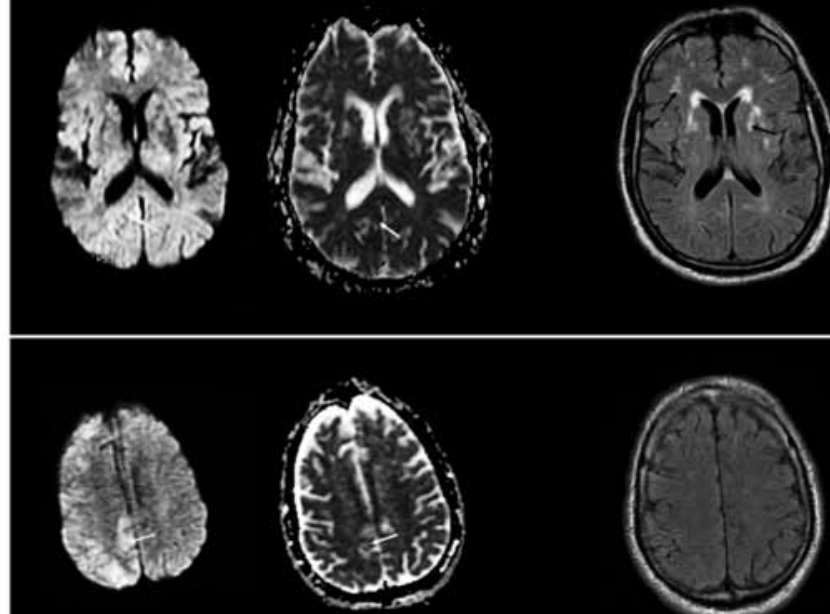

\section{DAY 7}

\section{DAY 20}
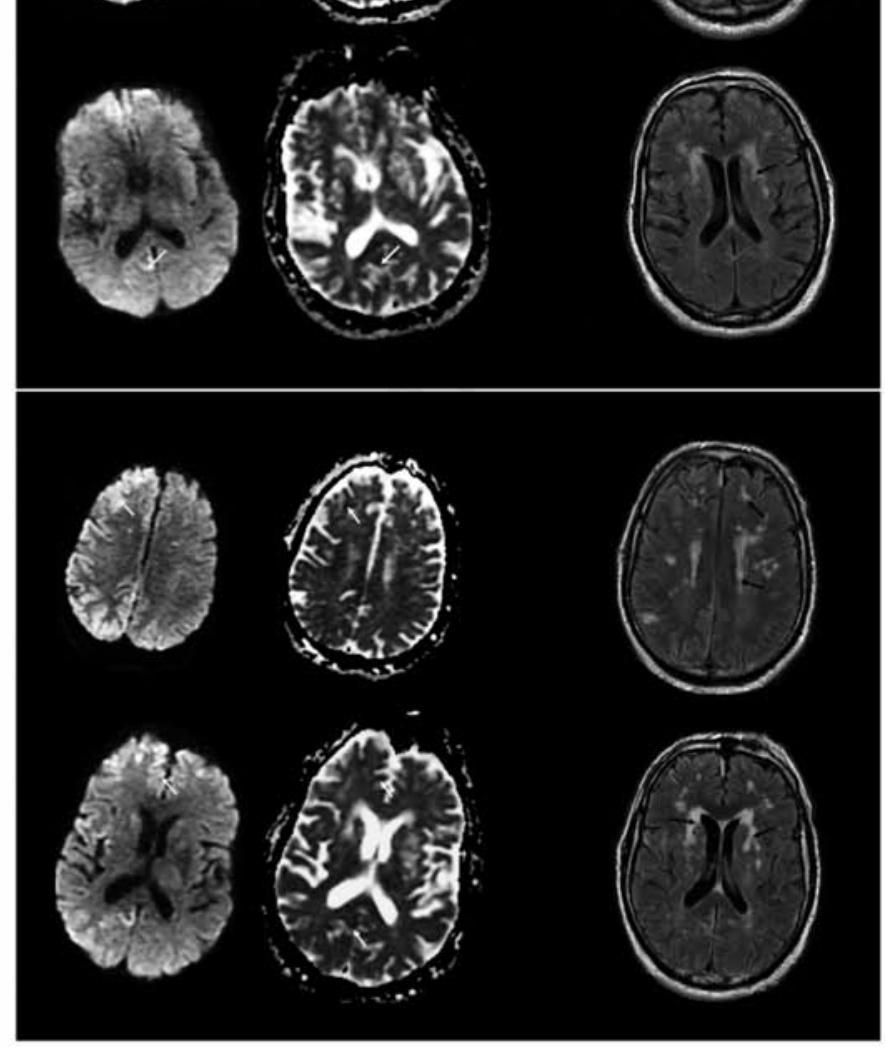

Figure 1. Brain magnetic resonance imaging on hospital days 1, 7, and 20 showed areas of restricted diffusion in the cerebral cortex. The cortical areas affected appeared hyperintense on DWI (first column) and hypointense on ADC (second column). Each ADC map in the second column corresponds to the DWI image in the first column $(b=1000)$. Restricted diffusion was not present in the basal ganglia and thalamus. Only a few of the cortical lesions were visible on FLAIR sequences (third column). Selected areas with clearcut signal abnormalities are indicated by an arrow: yellow for DWI hyperintensities, for ADC hypointensities, red for FLAIR sCJD hyperintensities, and black for FLAIR chronic microvascular changes. towards stroke and encephalitis. It was not until the twentieth hospital day that SCJD was suspected, when the EEG showed typical PSWCs.

Updated diagnostic criteria for sporadic Creutzfeldt-Jakob disease

The contemporary diagnosis of sCJD is largely based on the 1998 WHO criteria (Table 1). ${ }^{1}$ Using these criteria, a patient can be diagnosed with probable or possible sCJD. Unfortunately, strict reliance on WHO criteria may result in a failure to recognize sCJD because focal findings may predominate, $\operatorname{cog}$ nitive dysfunction may be absent or subtle, and the classic red flag for CJD - PSWCs - may not yet be present in the EEG. Some of these diagnostic pitfalls can be avoided by being familiar with the most current UCSF and MCC criteria.

The UCSF criteria differ from the WHO criteria by taking into account specific higher cortical signs (e.g. neglect, aphasia, apraxia, acalculia), by removing CSF 14-3-3 protein as a criterion, by adding MRI subcortical/cortical hyperintensities as criteria, and by expanding the definition of positive EEG to include periodic epileptiform discharges other than PSWCs (e.g. PLEDs). ${ }^{2}$

The MCC criteria differ from the WHO and UCSF criteria by putting less emphasis on cognitive dysfunction, allowing one to diagnose probable sCJD in a cognitively intact person with visual/cerebellar disturbances, pyramidal/extrapyramidal signs, and at least one positive test result. The MCC criteria also differ from the other two sets by including EEG, CSF 14-3-3, and MRI findings as positive laboratory tests; however, it retains the original WHO definition of a positive EEG as one that shows typical PSWCs. ${ }^{3}$

\section{Diagnosing sporadic Creutzfeldt-} Jakob disease remains a challenge

The differential diagnosis of rapidly progressive dementia is broad. ${ }^{5}$ Myoclonus helps narrow down the differential diagnosis to some extent. However, cognitive dysfunction may be subtle and myoclonus may be absent in the early stages of sCJD. Furthermore, EPC is not typically viewed as myoclonus when, in fact, EPC is just another name for focal myoclonic status epilepticus. ${ }^{6}$ The lack of such awareness can delay the diagnosis of sCJD in a patient with EPC as an early manifestation.

A wide range of focal/ateralized manifestations have been reported in patients with sCJD. Examples of foca//lateralized neurological signs/symptoms in sCJD are: i) focal higher cortical deficits, such as aphasia, ${ }^{7}$ alexia without agraphia, ${ }^{8}$ and alien limb phenomenon; ${ }^{9}$ ii) movement disorders other than myoclonus, ${ }^{10}$ focal dystonia, ${ }^{11}$ and cerebellar ataxia;12 iii) visual abnormalities such as 


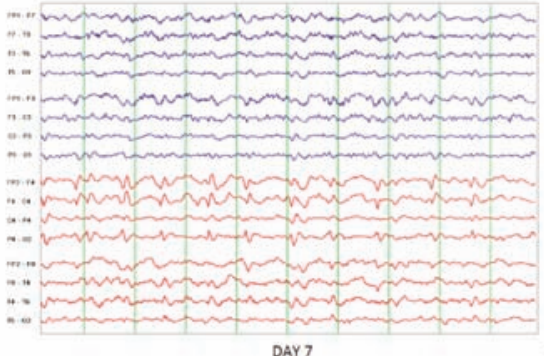

DAY 7

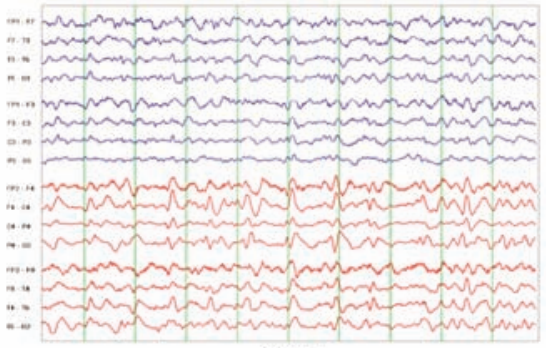

DAY 14

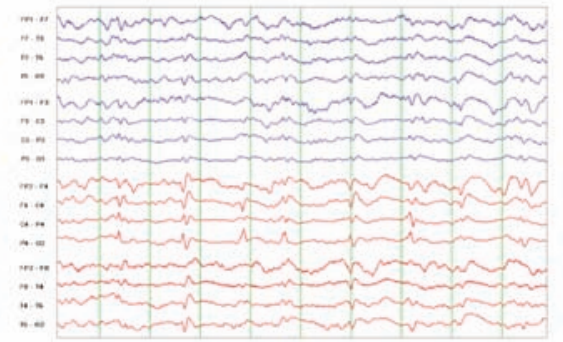

DAY 10

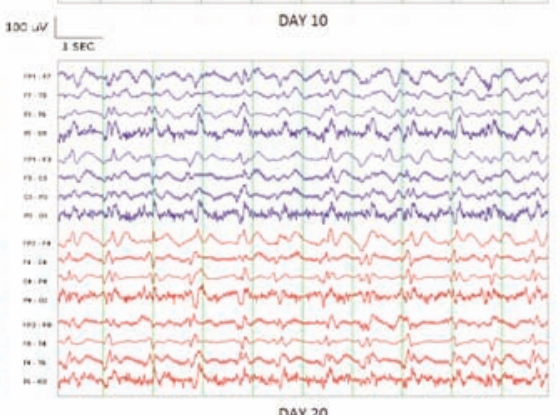

DAY 20

Figure 2. Scalp EEG on hospital days 7, 10, and 14 showed right hemispheric PLEDs in the form of sharp and slow waves with a right centroparietal maximum and a discharge rate of about $1 /$ second. Some sharp waves were time-locked to the patient's myoclonic jerks. The background EEG was slow and attenuated on both sides. The PLEDs increased in amplitude and became broader in distribution. On day 20, the EEG showed bihemispheric PSWCs (right lower tracing) alerting the physicians to the possibility of $s C J D$. For all tracings: screen sensitivity $=5 \mu \mathrm{V} / \mathrm{mm}$, timebase $=1$ second $/$ division. EEG tracings from the left side are in blue and tracings from the right side are in red.

hemianopia, diplopia, 13 and the well-known Heidenhain phenotype; 14 motor weakness, such as hemiparesis and monoparesis; $; 5,16$ and focal seizure activity, particularly EPC.17-19 In many of these cases, the focal deficit is the initial manifestation of SCJD, and in some, the focal symptoms are acute in onset leading to an incorrect diagnosis of stroke. 15,20 There are also post-mortem neuropathological studies of sCJD with focal/lateralized manifestations in which the distribution of spongiform changes and neuronal loss in the brain correlate well with the clinical picture. 21,22

Although generalized PSWCs strongly indicate $\mathrm{SCJD}, 23$ the EEG in the early stages of sCJD often shows non-specific background slowing, frontal intermittent rhythmic delta activity, or foca//lateralized abnormalities such as PLEDs.24,25 It may take days or weeks before classic PSWCs appear on the EEG. Some sCJD patients with PLEDs also manifest EPC. Because stroke is the most common condition associated with PLEDs, the combination of PLEDs and focal deficits will usually encourage a diagnosis of stroke. PLEDs can also steer the diagnosis towards encephalitis, especially herpes encephalitis. In the case presented, the initial diagnosis was stroke with encephalitis considered a possibility later.

CSF 14-3-3 protein assay has a relatively low specificity and a modest sensitivity for diagnosing sCJD. ${ }^{26}$ It was in fact eliminated as a diagnostic criterion from the UCSF criteria set. ${ }^{2}$ Nevertheless, until a highly specific sCJD biomarker becomes available, CSF 14-3-3 protein determination remains useful in the workup of a patient with suspected SCJD especially if there is no alternative diagnosis. ${ }^{27}$ As stated before, MRI findings have been incorporated in the UCSF and MCC criteria for probable sCJD.2,3 The MRI lesions of sCJD often appear as areas of restricted diffusion (DWI hyperintensities and ADC hypointensities) and FLAIR hyperintensities in the cerebral cortex, basal ganglia, and/or thalamus. ${ }^{28}$ The lesions are often multifocal and bilateral but, early in the stage of SCJD, they can be lateralized or unilateral (as in our patient) and lead to an incorrect diagnosis of cerebral infarction. ${ }^{29} \mathrm{DWI}$ with $\mathrm{ADC}$ proved to be more sensitive than FLAIR imaging in tracking the SCJD cortical lesions of our patient. This is consistent with the results of the MCC study which showed a DWI sensitivity of $85.2 \%$ and a FLAIR sensitivity of $74.9 \%$ if the minimum lesion for sCJD detection is defined as the presence of hyperintensities in at least two cortical areas and/or in the basal ganglia. ${ }^{3}$ A recently published multicenter study comparing DWI and FLAIR also concluded that DWI is more sensi- tive than FLAIR for detecting sCJD. 30

The polymorphism of the prion protein gene at codon 129 and the pattern of prion protein migration on Western blot provide a basis for classifying sCJD into six molecular subtypes: MM1, MM2, MV1, MV2, VV1 and VV2. ${ }^{31}$ In all of these sCJD subtypes, the damage occurs in the cerebral cortex, basal ganglia, and/or thalamus. However, certain molecular subtypes will correlate well with certain lesion patterns in the MRI. ${ }^{32}$ For example, the absence of DWI/FLAIR lesions in the basal ganglia and thalamus (as in the case presented) points towards the VV2 molecular subtype. Unfortunately, molecular typing was not performed in our patient.

\section{Beyond diagnostic criteria}

In refining and expanding the WHO criteria, the UCSF and MCC criteria will reduce the chances of a false negative diagnosis of SCJD. Unfortunately, regardless of the criteria set used, sCJD with early focal clinical manifestations, sCJD with atypical features, and SCJD in its early stages with no diagnostic EEG and MRI changes can still escape early diagnosis. This is a frightening scenario for a disease that is transmissible, incurable, and $100 \%$ fatal. We have identified a number of caveats to the available criteria to remind physicians that other measureable, although traditionally atypical, signs should not immediately rule out a diagnosis of CJD (Table 2). Physicians who employ repeated measures of key diagnostic tests and those who are willing to consider a wider constellation of signs will have the greatest success in providing timely diagnosis and avoiding potential transmission of CJD to health care providers and patient contacts.

\section{Consent}

This manuscript presents a single case report with no systematic design to answer a research question. This project was determined to be exempt from oversight by the LSUHSC-NO IRB.

\section{References}

1. WHO Consultation on Global Surveillance, Diagnosis and Therapy of Human Transmissible Spongiform Encephalopathies. WHO/EMC/ZDI/98.9 Geneva: WHO; 1998.

2. UCSF 2007 criteria for probable sporadic Jakob-Creutzfeldt Disease: UCSF memory and aging center. Available from: http://memory.ucsf.edu/cjd/medical/cjddiagnosis/criteria/single.

3. Zerr I, Kallenberg K, Summers DM, et al. Updated clinical diagnostic criteria for 
sporadic Creutzfeldt-Jakob disease. Brain 2009;132:2659-68.

4. Budka H. Neuropathology of prion diseases. Br Med Bull 2003;66:121-30.

5. Geschwind MD, Shu H, Haman A, et al. Rapidly progressive dementia. Ann Neurol 2008;64:97-108.

6. Mader EC Jr, Olejniczak PW. Epilepsy syndromes. In: Bruce J. Fisch, ed. Epilepsy and intensive care monitoring: principles and practice. New York; Demos Publishing; 2010. pp 119-150.

7. Kirk A, Ang LC. Unilateral CreutzfeldtJakob disease presenting as rapidly progressive aphasia. Can J Neurol Sci 1994; 21:350-2.

8. Adair JC, Cooke N, Jankovic J. Alexia without agraphia in Creutzfeldt-Jakob disease. J Neurol Sci 2007;263:208-10.

9. Rubin M, Graff-Radford J, Boeve B, et al. The alien limb phenomenon and Creutzfeldt-Jakob disease. Parkinsonism Relat Disord 2012 May 8. [Epub ahead of print].

10. Maltête D, Guyant-Maréchal L, Mihout B, Hannequin D. Movement disorders and Creutzfeldt-Jakob disease: a review. Parkinsonism Relat Disord 2006;12:65-71.

11. Hellmann MA, Melamed E. Focal dystonia as the presenting sign in CreutzfeldtJakob disease. Mov Disord 2002;17:1097-8.

12. Laverse E, Shah S, Mavra M. Sporadic Creutzfeldt-Jakob disease: early signs and pre-mortem diagnosis. BMJ Case Rep 2009;2009:piibcr07.2009.2105.

13. Proulx AA, Strong MJ, Nicolle DA. Creutzfeldt-Jakob disease presenting with visual manifestations. Can J Ophthalmol 2008;43:591-5.

14. Cooper SA, Murray KL, Heath CA, et al. Isolated visual symptoms at onset in spo- radic Creutzfeldt-Jakob disease: the clinical phenotype of the Heidenhain variant. Br J 0phthalmol 2005;89:1341-2.

15. Ko KF, Lau WY, Cheng WK, et al. Creutzfeldt-Jakob disease with initial right hemiparesis masquerading as a stroke. Hong Kong Med J 2010;16:487-8.

16. Obi T, Takatsu M, Kitamoto T, et al. [A case of Creutzfeldt-Jakob disease (CJD) started with monoparesis of the left arm]. Rinsho Shinkeigaku 1996;36:1245-8. [Article in Japanese].

17. Lee K, Haight E, Olejniczak P. Epilepsia partialis continua in Creutzfeldt-Jakob disease. Acta Neurol Scand 2000;102:398402.

18. Parry J, Tuch P, Knezevic W, Fabian V. Creutzfeldt-Jakob syndrome presenting as epilepsia partialis continua. J Clin Neurosci 2001;8:266-8.

19. Taskiranis Taskiran A, Tezer FI, Saygi S. Epilepsia partialis continua as the presenting symptom in probable sporadic Creutzfeldt-Jakob disease. Epileptic Disord 2011;13:82-7.

20. Hirst CL. Sporadic Creutzfeldt-Jakob disease presenting as a stroke mimic. $\mathrm{Br} \mathrm{J}$ Hosp Med (Lond) 2011;72:590-1.

21. Yamanouchi H, Budka H, Vass K Sr. Unilateral Creutzfeldt-Jakob disease. Neurology 1986;36:1517-20.

22. Heye N, Henkes H, Hansen ML, Gosztonyi G. Focal-unilateral accentuation of changes observed in the early stage of Creutzfeldt-Jakob disease. J Neurol Sci 1990;95:105-10.

23. Steinhoff BJ, Zerr I, Glatting M, et al. Diagnostic value of periodic complexes in Creutzfeldt-Jakob disease. Ann Neurol 2004;56:702-8.

24. Wieser HG, Schindler K, Zumsteg D. EEG in Creutzfeldt-Jakob disease. Clin Neurophysiol 2006;117:935-51.

25. Hansen HC, Zschocke S, Stürenburg HJ, Kunze K. Clinical changes and EEG patterns preceding the onset of periodic sharp wave complexes in Creutzfeldt-Jakob disease. Acta Neurol Scand 1998;97:99-106.

26. Geschwind MD, Martindale J, Miller D, et al. Challenging the clinical utility of the 14-3-3 protein for the diagnosis of sporadic Creutzfeldt-Jakob disease. Arch Neurol 2003;6:813-6.

27. Skinningsrud A, Stenset V, Gundersen AS, Fladby T. Cerebrospinal fluid markers in Creutzfeldt-Jakob disease. Cerebrospinal Fluid Res 2008;27:14.

28. Young GS, Geschwind MD, Fischbein NJ, et al. Diffusion-weighted and fluid-attenuated inversion recovery imaging in Creutzfeldt-Jakob disease: high sensitivity and specificity for diagnosis. AJNR Am J Neuroradiol 2005;26:1551-62.

29. Fujita K, Harada M, Sasaki M, et al. Multicentre multiobserver study of diffusion-weighted and fluid-attenuated inversion recovery MRI for the diagnosis of sporadic Creutzfeldt-Jakob disease: a reliability and agreement study. BMJ Open 2012;2:e000649.

30. Bavis J, Reynolds P, Tegeler C, Clark P. Asymmetric neuroimaging in CreutzfeldtJakob disease: a ruse. J Neuroimaging 2003;13:376-9.

31. Letourneau-Guillon L, Wada R, Kucharczyk W. Imaging of prion diseases. J Magn Reson Imaging 2012;35:998-1012.

32. Meissner B, Kallenberg K, Sanchez-Juan P, et al. MRI lesion profiles in sporadic Creutzfeldt-Jakob disease. Neurology 2009;72:1994-2001. 